\title{
COMMENTS ON P. POTIER'S SCIENTIFIC ACHIEVEMENTS
}

The scientific carreer of Pierre Potier began in 1957 with the preparation of his Ph.D. under the supervision of Professors Maurice-Marie Janot and Jean Le Men, in Paris. He worked on the pseudoalkaloids present in the seeds of Honesty (Lunaria biennis, Moench, Cruciferae).

In collaboration with Peter Pauson and Peter Bladon (Glasgow) he was able to demonstrate, for the first time, the presence of spermidine as a part of the molecule of lunarine. Spermidine was so far considered as a typical "animal product". Degradation of lunarine ultimately led to the establishment of its formula which was confirmed by crystallographic studies. The possible biological implications of this research have not yet been fully explored : in lunarine, spermidine is "locked" by two p-hydroxycinnamic units as a bisamide formed with the two primary amine functions of spermidine.

This bis-amide (or equivalent bioprecursor) then undergoes an oxidative coupling reaction (analogous to that leading to Pummerer's ketone and related systems) leading to lunarine and related products.

The presence of such polyamine derivatives (putrescine, spermidine, spermine, etc.) is apparently common to all seeds.

These compounds certainly play an important role during the first steps of germination of seeds by delivering, after the action of some peptidases, both the polyamine and phenolic acids necessary for plant development (publication $\mathrm{N}^{\circ} 1$ and related papers).

Some plants are known to contain "steroidal alkaloids" or, better, aminosteroids. Their biosynthesis most probably consists of a transamination reaction of the corresponding precursors. Applying this simple hypothesis, Pierre Potier was able to isolate from Paravallaris microphylla Pitard (Apocynaceae) progesterone and related ketonic compounds for the first time in the plant kingdom. Odd but not at all unexpected, this is a prime example of chemical convergence! 
In 1965, a number of hypotheses were put forward to explain the biosynthesis of some complex natural products, in particular the thoroughly studied indole alkaloids.

Pierre Potier, together with Alan Battersby (U.K.) was able to isolate and elucidate the chemical structure of a crucial intermediate in the biosynthesis of isoquinoline (and, by extension, of indole) alkaloids. This discovery opened the way to research in elucidating the biosynthesis of this very important class of natural compounds.

The well-known Polonovski reaction discovered in the late 1920's by the Polonovskis (chemist and biochemist) consists of treating N-oxides with various acid anhydrides. Pierre Potier discovered, in 1965, an interesting modification of this reaction by replacing acetic anhydride of the genuine Polonovski-reaction by trifluoroacetic anhydride. This change in the reaction conditions entirely modified the course of the reaction : elimination and fragmentation reactions could take place depending on the stereoelectronic characteristics of the reactants.

This new reaction (described recently in "Organic Reactions") was later termed the "Polonovski-Potier reaction".

It opened the way to the preparation of a number of iminium ions used in the synthesis of various natural products. Those derived from the indole nucleus were particularly amenable to the application of this new reaction. A number of syntheses of complex indole alkaloids were successfully achieved.

In particular, the biomimetic synthesis of the antitumour alkaloids of the vinblastine group was achieved for the first time.

An entirely new class of alkaloids of the vinblastine group was discovered using the Polonovski-Potier reaction : the nor-5'-anhydrovinblastine class. Among these is NAVELBINE, which now constitutes one of the best drugs used in the treatment of "non-small cell" lung tumours, breast cancers, ovarian cancers, etc. 
Another completely new natural compound was isolated from a marine sponge collected in New Caledonia $[($ Pseudoaxinyssa cantharella, n. sp., (Axinellideae)] : girolline, an amino-2-imidazole. This compound exhibited a very large spectrum of antitumour activity and was evaluated in phase I clinical trials. However, these trials were stopped due to unfavorable effects on blood pressure. Research is nevertheless continuing on this type of compound as it possesses an original mode of biological action.

The biological target of NAVELBINE, (vide supra) as well as other naturally-occurring compounds (vinblastine group, colchicine, podophyllotoxin, etc.) is a ubiquitous protein called tubulin. This protein is known to polymerise (GTP-dependent process) into microtubules which ultimately constitute the spindle necessary for cell division. Based on this set of biologically important events, a screening test was developed in Pierre Potier's laboratories which enabled selection of new antitumour compounds. The correlation between results obtained using this very simple test and the actual activity of the selected compounds as antitumour drugs is quite good.

An important application of this strategy has been the discovery of a new antitumour drug, TAXOTERE®, which has been launched worldwide by Rhône-Poulenc Rorer.

TAXOTERE is a compound belonging to the same class of natural products as Taxol. Taxol, a diterpene isolated in 1967 by WANI and WALL in the US, has revealed very interesting antitumour properties, in particular in the treatment of advanced ovarian cancers as well as other tumour types. Unfortunately, taxol is present, in low concentrations, in the trunk bark of the Pacific yew tree in the US. This situation has led the National Cancer Institute and other people (in industry) to program the collection of several tons of yew trunk bark which constitutes a very serious ecological problem.

As early as 1980 Pierre Potier's group began research in this field. The first target was to try to find a new source of taxol which would not necessitate the destruction of thousands of yew trees. They found that a relatively abundant precursor of taxol was present in the leaves (needles) of the European yew tree [Taxus baccata, L. (Taxaceae)] : 10-deacetyl baccatin III. This compound can be rather easily isolated and used as a starting material for the preparation of either TAXOTERE, or Taxol or related active 
compounds.

This discovery is important as it opens the way to a number of chemical, biochemical and therapeutic studies concerning this very interesting type of anticancer drug.

All these compounds can be prepared in quantities limited only by the collection of sufficient yew tree leaves, a rapidly renewable resource.

Pierre Potier has also been involved in the study or search for new compounds interacting with the benzodiazepine receptor, in relation with the study of the possible role of endogenous substances in anxiety, memory processes and immunology, etc.

With D. H. R. Barton he has studied the reductive radical decarboxylation of amimo acids and peptides.

His current research is at the moment concerned with the chemistry, biochemistry and possible therapeutic applications of nitric oxide (NO) (and related compounds). This very simple chemical compound plays a very important role in biological systems as a universal mediator. A number of exciting discoveries have to be expected in the near future in this field of research, particularly in the chemotherapy of cancer and parasitic diseases.

Recently, Pierre Potier has launched a series of research in the field of diabetes type II. A number of interesting results have been obtained. This disease attains ca 200 Millions peoples in the world and is sharply progressing. 